\title{
Overview of Technique Aspects in Relay Satellite System
}

\author{
Yuan Gao ${ }^{1,3, a}$, Hong Ao, b , Quan Zhou ${ }^{1, c}$, Weigui Zhou ${ }^{1, d}$ and $\mathrm{Yi} \mathrm{Li}^{2, e^{*}}$ \\ ${ }^{1}$ Xichang Satellite Launch Center, Sichuan, China \\ ${ }^{2}$ The High School Affiliated to Renmin University of China, Beijing, China \\ ${ }^{3}$ China Defense Science and Technology Information Center, Beijing, China \\ ayuangao08@gmail.com, baohong76@aliyun.com, czhouquanxslc@foxmail.com, \\ wgzhou@nudt.edu.cn, ${ }^{\mathrm{e}}$ liyi@rdfz.cn \\ * The corresponding author
}

Keywords: Satellite; Data relay system; Overview; Technique

\begin{abstract}
Data relay satellites as an important part of space-based communication systems, greatly improving the user's satellite coverage, reducing the number of ground stations cloth, cost savings, and is one of the major space-faring countries focused on building space systems. Currently, the United States, Russia, Europe and Japan have developed their own data relay satellite system in space and terrestrial systems to upgrade at the same time, a small satellite constellation, equipped with flexible form loads like laser communications, software radio, networking and other advanced technology development and deployment of next generation active satellite systems, and planning to build a relay across the solar system with planets satellite relay satellite.
\end{abstract}

\section{Development of Data Relay Satellite in Main Countries}

Status of USA. American TDRSS system, also known as space-based network, the monitoring network is one of the three NASA (National Aeronautics and Space Administration, NASA), mainly for the United States military and civilian LEO satellites, the International Space Station, research aircraft and balloons, carrying rockets and other users with $\mathrm{S}, \mathrm{Ku}, \mathrm{Ka}$-band monitoring and control information and business data relay. By the end of 2014, the United States has a total of 11 launch third generation TDRS satellites in orbit serving has 9, two sites in the construction of three ground terminal station, consisting of a complete satellite system. According to NASA "Space Communications and Navigation" (Space Communications and Navigation, SCaN) plan [1], the main task facing the recent demand for space-based network communication is reliable unmanned lunar probes require high-speed data transmission and manned missions needed, high-speed backbone link, nearly continuous coverage and seamless support for user tasks. Therefore, TDRSS construction of key recent years are: launch the third generation of satellites; use of 30 years of ground system updates and upgrades; New Blossom [2] ground station to meet future aerospace needs protection.

SGSS plan also includes the establishment of Maryland Blossom corner a new terminal station, east extended coverage of space-based network, to provide launch support for the expendable launch vehicle, known as the Space Network Expansion terminal station, the station will be built two main tasks and configure new ground antenna communications electronics. SGSS innovation system is a "pool" structure to manage the device. The current space-based network ground terminal station a "chimney" structure, the signal is sent to a particular series of devices through TDRS satellites. Although the device has redundant, but you cannot switch to another TDRS satellites to support users. This structure limits the flexibility to increase the number of devices on each end of the ground, because every single TDRS satellite itself needs a master / standby ground equipment. The SGSS system uses the "pool" structure, and every single TDRS satellite dedicated little device, the user can choose to protect equipment from the free pool of resources, returned to the pool after performing the task for the next use, each ground terminal station can using the same pool. "Pool" type structure 
reduces the amount of equipment to improve the structural flexibility and hardware utilization efficiency.

SGSS program was started in July 2014, the original plan in June 2017 for acceptance review, but the plan has been a predetermined time lag of 2 years (expected Acceptance Review has been delayed until May 2019). Due to cost more than the budget 30\%, NASA had to cancel some of the contents, including some backup capabilities and simulation capabilities, so SGSS system capacity decreased.

SBRS from the following three aspects of the future architecture of the study [3] [4].

User Needs and Business Performance. According to 2020 - Space Mission (especially manned space) needs to determine the satellite's orbit coverage level and the relationship between the scheduling of on-demand service, latency, data rate and amount of data, business cycle, security planned for 2040 requirements.

Technical / Physical Structure. Assessment techniques include optical communications, microwave communications, satellite links between the millimeter wave phased array antennas, disruption tolerant networking, networking space, cognitive and adaptive protocols, and software radio technology. The physical structure of the satellite system can take many forms, including dedicated satellite (similar to the existing TDRS satellites), mounted on the load as boarding commercial satellites or other government satellites, have a small satellite constellation, and combinations thereof in the form of distributed capabilities. Structure of the program will be considered World Network integration.

Procurement and Management. Including public-private partnerships, business purely commercial, shared ownership with other government agencies, NASA dedicated / proprietary systems. 2014, NASA Glenn Research Center proposed based on a small satellite in geosynchronous orbit data relay satellite program "Small space-based satellites" (Small Space Based Satellite, SSBS). SSBS not only to solve the needs of Earth observation mission, but also to address the needs of an asteroid and Mars manned space mission in 2030, so the design of satellites with SSBS support synchronous orbit (Geosynchronous Earth Orbit, GEO) and the ability to link the above tasks . Satellite will be put into use in 2022, by the "Falcon 9" rocket to launch an arrow Samsung ways to reduce launch costs. Dual-band satellite antenna can be above and below the GEO satellite communication with the user, and will retain and enhance the $\mathrm{S}$ and Ka single site business, satellite-ground link with the current TDRS same (Ku-band), an increase of optical communications options, and now there TDRS provide communications relay and tracking the same business. SSBS is the first US manned probe to take the relay system, the future of lunar relay satellite, the Mars Relay Satellite will SBSS reference design, they are basically the same function, but according to mission requirements and estimated fuel consumption, differ in redundancy and frequency used.

Status of Europe. ESA (European Space Agency) [5] launched in 2001, although the first generation of data relay satellite Artemis, and put into use in 2003, but the only satellite satellites technology test satellites, and achieve lifetime in 2010. With the "Global Monitoring for Environment and Security" (GMES) Implementation Plan EU / ESA joint, the estimated amount of data every day from space to terrestrial transmission will reach $6 \mathrm{~TB}$, current European Space communication system cannot meet the need. In order to achieve strategic independence in Europe, ESA from 2008 proposed construction of the "European Data Relay Satellite" (EDRS) system.

EDRS program after several years of development and evolution, and ultimately determine system with two synchronous orbit node and ground system consisting of ground. Two spatial nodes which is equipped with a laser communications payload (EDRS-A), and the other is a dedicated Data Relay Satellite (EDRS-C), covering only two nodes in Europe in the European commercial communications satellites in Eutelsat 9B. EDRS-A between the laser and provide two kinds of Ka-band satellite communication links, laser back to the data rate up to $1.8 \mathrm{~Gb} / \mathrm{s}$; Ka-band return to up to $300 \mathrm{Mb} / \mathrm{s}$; EDRS - C only laser link, index and EDRS - A same. According to schedule ESA, EDRS-A launch was in January 2016, EDRS-C satellite will be launched in early 2017. The two satellites are 15 years of life.

EDRS first laser communication user is "Sentinel" series of satellites. April 3, 2014, the first satellite "sentinels 1A" launch 2015 summer officially started on a loop track communication 20 min. 
The first Ka-band user is the International Space Station, "Columbus" cabin for real-time data on the ground to test satellite transmission in 2016 enabled. EDRS is a key market for future US Department of Defense and its UAV fleet. UAV fleet will produce large amounts of video data stream, and radio frequency (currently using Ku-band, Ka-band for future use) compared to laser links can transmit data more quickly. By design, a satellite with four UAV communication speeds up to $1.8 \mathrm{~GB} / \mathrm{s}$, the data capacity per aircraft per hour $1.6 \mathrm{~Tb}, 4$ planes altogether $6.4 \mathrm{~Tb}$. ESA and Airbus plans to expand the system EDRS third geosynchronous orbit node, which will be located in the Western Pacific Ocean, it is likely to use a piggyback payload form, in order to achieve global coverage. Plan named "Global Network" (GlobeNet), the final plan will be determined 2015 to 2016, according to current plans, the use of two laser communication terminals (Laser Communication Terminal, LCT) terminal, including authentication GEO - GEO satellite link between Road, satellite scheduled for launch in 2020. Traffic considering the continuous improvement of secure communication requirements, such as direct command and control of the secret payload and more redundancy requirements and demand for UAVs sensitive data relay. LCT interoperability interface in favor of international cooperation, has been included in the International Advisory Committee for Space Data Systems (Consultative Committee for Space Data Systems, CCSDS) standardization program as input.

Status of Japan. Japan launched in $2002 \mathrm{ka}$ band satellite data relay test satellite (Data Relay Test Satellite, DRTS), is still running, the communication rate of $240 \mathrm{Mb} / \mathrm{s}$. With the Japanese earth observation satellite with high accuracy the development, the surge in demand for data transmission. In 2008, Japan launched the development of the next generation of laser communications relay satellite terminal, the design goal is to make the next generation of communication speed relay satellite between the satellite and the user reaches 2.5 GB / s. In 2011, the Japanese Space Agency (JAXA) presented two next-generation data relay satellite system program, a use of optical communications technology, forward / reverse data rate of $2.5 / 1.2 \mathrm{~Gb} / \mathrm{s}$; the other did not use optical communication technology, DRTS only on the basis of the increase, called "DRTS-2", the entire program is similar to the DRTS, just increase the data rate Ka-band for the reverse link [6].

In 2014, Japan announced plans to launch "optical data relay satellite" in 2019, the use of optical communication to communicate with RF combination of programs, the use of lasers and S / Ka-band and "advanced optical satellites" and other low-orbiting satellites to relay communications, data transmitted to the Tsukuba station and Tateyama station, optical communication speeds up to $1.8 \mathrm{~Gb} /$ $\mathrm{s}$, the life of a tentative 10 to 15 years. JAXA plans to complete the same year "advanced optical satellites" and "optical data relay satellite" launch, land and sea observation to apply real-time high-resolution observation and high frequency of disasters.

\section{Suggestions to China}

Improve Data Transmission Rate Using Laser Communication Technology. As mentioned above, laser communication technology can greatly improve the data transfer rate, due to the current technology is not mature enough, and from the practical application is still lagging behind. National and regional programs from next-generation data relay satellite system perspective, Russia, Europe, the United States [7] and Japan are considering laser communications technology. Russia has joined the active satellite laser communication channel. Europe has an absolute advantage in laser communications, its next-generation system solutions no matter what changes, laser communication technology determination never changed. In recent years, the literature shows that Europe laser communications technology and equipment development aspects of outstanding results, with the approaching launch date, laser communication technology put into practical use around the corner. Japan and the United States are given two options using only RF system and increasing the laser communication system, the Japanese chose the laser communication solutions. Although the United States has not been finalized, but in recent years are also actively carry out demonstration and communication technology in the NASA laser technology roadmap. Laser communications relay satellite technology is the inevitable trend of the future. 
Satellite Platform Diversification. The United States in 2013 has issued "invulnerability and shredded Space Architecture" white paper and the "future military satellite communications" report for the increasingly confrontational space environment, put forward a "shredded" thought the large satellites of functional decomposition to small and boarding satellite payload to complete [8], in order to improve the anti-space systems survivability and affordability. This idea has been embodied in the United States to build the next generation of aerospace systems in the system. Currently, the United States put forward the next generation of reconnaissance satellites, early warning satellites, communication satellites, meteorological satellites are used in the form of small satellite platform or carrying loads. This idea is also reflected in the design of the relay satellite system. US relay satellite program uses the form of small satellite constellation, Europe and dedicated satellite carrying the load combination of form, so that the relay satellite system to build a more flexible, but also improve the flexibility of the satellite system.

Use Networking Technology to Build a Satellite Network. As mentioned earlier, the United States, Japan, utilize relay satellite test conducted DTN technology [9] and software radio technology validation, to lay the foundation for the future to build a satellite network interconnection. Europe proposes to increase the first three EDRS satellites using laser communication link Construction ISLs between a relay satellites, then relay satellite constellation will form a network of networks. TDRS program by the United States have the ability to communicate with the GEO spacecraft above[10-13], the United States will in future Moon, Mars construction Relay Satellite System, which relay satellite relay satellite earth together, will constitute the use of networking technology interplanetary network backbone.

\section{Summary}

In this work, Data Relay Satellite System as an important part of the space-based monitoring and control system, by the national attention. China has also set up their own data relay satellite system, and is developing a second-generation system. Looking at other countries and regions to design the next generation of satellite systems, whether in the form of system construction or application of new technologies have changed greatly. We should be combined with national conditions and future manned and unmanned space missions demand, the use of laser communication, DTN network, software radio and other advanced technology, fully tap the potential relay satellite system, to build their own Relay Satellite System . In the game space increasingly fierce new forms, how to set up anti-jamming, anti-destroy, elastic anti-satellite system worth considering cyber-attacks.

\section{Acknowledgements}

This work is funded by China's 973 project under grant of 2012CB316002 and China's 863 project under grant of 2013AA013603, 2012AA011402, National Natural Science Foundation of China(61201192), The Open Research Fund of National Mobile Communications Research Laboratory, Southeast University (2012D02); International Science and Technology Cooperation Program(2012DFG12010); National S \& T Major Project (2013ZX03001024-004) ,Operation Agreement Between Tsinghua University and Ericsson, Qualcomm Innovation Fellowship, whose funding support is gratefully acknowledgment. The author would also like to thank all the reviewers, their suggestions help improve the work a lot.

\section{References}

[1] Yi Xu, Shiwen Mao, and Xin Su, "Interference alignment improves the capacity of OFDMA systems," IEEE Transactions on Vehicular Technology, vol.65, no.2, pp.756-767, Feb. 2016. DOI: 10.1109/TVT.2015.2402191. 
[2] Gao Y, Zhou W, Ao H, Chu J, Zhou Q, Zhou B, Wang K, Li Y, Xue P. A Novel Optimal Joint Resource Allocation Method in Cooperative Multicarrier Networks: Theory and Practice. Sensors. 2016; 16(4):522

[3] K. Fall, "A delay-tolerant network architecture for challenged Internet," in Proc. SIGCOMM, Karlsruhe, Germany, pp. 27-34.

[4] F. Warthman, "Delay-tolerant networks (DTNs): A tutorial," Warthman Associates, Palo Alto, CA, 2003 [Online]. Available: http://www.ipnsig.org/reports/DTN_Tutorial11.pdf

[5] V. Cerf, S. Burleigh, A. Hooke, L. Torgerson, R. Durst, K. Scott, K. Fall, and H. Weiss, "Delay-tolerant networking architecture," IETF, RFC 4838, Apr. 2007 [Online]. Available: http://www.ietf.org/rfc/rfc4838.txt

[6] S. Burleigh and K. Scott, "Bundle protocol specification," IETF, RFC 5050, Nov. 2007 [Online]. Available: http://www.ietf.org/rfc/rfc5050.txt

[7] M. Demmer and J. Ott, "Delay tolerant networking TCP convergence layer protocol," IETF DTNRG IRTF Research Group,<draft-irtf-dtnrg-tcp-clayer-02.txt> (work in progress), Nov. 2008[Online]. Available: http://www.ietf.org/internet-drafts/draft-irtfdtnrg-tcp-clayer-02.txt

[8] J. Postel, “User datagram protocol,” IETF, RFC 768, Aug. 1980.

[9] L. Wood, W. M. Eddy, W. Ivancic, J. McKim, and C. Jackson, "Saratoga: A delay-tolerant networking convergence layer with efficient link utilization," in Proc. IWSSC, Salzburg, Austria, Sep. 2007,pp. 168-172.

[10]H. Cruickshank, P. Pillai, M. Noisternig, and S. Iyengart, Security Requirements for the Unidirectional Lightweight Encapsulation (ULE) Protocol, Internet RFC 5458, May 2009.

[11] S. Bellovin, BProbable plaintext cryptanalysis of the IP security protocols, in Proc. IEEE Symp. Netw. Distrib. Syst. Security, San Diego, CA, 1997, pp. 52-59.

[12] J. Sing and B. Soh, BA critical analysis of multi-layer IP security protocol, in Proc. 3rd Int. Conf. Inf. Technol. Appl., Sydney, Australia, 2005, pp. 683-688.

[13] Y. Zhang, BA multilayer IP security protocol for TCP performance enhancement in wireless networks, IEEE J. Sel. Areas Commun., vol. 22, no. 4, May 2004. 